\title{
Current situation and consideration on the enhanced recovery protocols in lung cancer surgery
}

\author{
Shuangjiang Li, Guowei Che, Cheng Shen, Kun Zhou \\ Department of Thoracic Surgery, West China Hospital, Sichuan University, Chengdu 610041, China \\ Correspondence to: Guowei Che. Department of Thoracic Surgery, West China Hospital, Sichuan University, Guoxue Alley No. 37, Chengdu 610041, \\ China. Email: guowei_che@foxmail.com. \\ Provenance: This is an invited Editorial commissioned by the Section Editor Shuangjiang Li (Department of Thoracic Surgery and West China \\ Medical Center, West China Hospital, Sichuan University, Chengdu, China). \\ Comment on: Rogers LJ, Bleetman D, Messenger DE, et al. The impact of enhanced recovery after surgery (ERAS) protocol compliance on morbidity \\ from resection for primary lung cancer. J Thorac Cardiovasc Surg 2018;155:1843-52.
}

Submitted Jun 26, 2018. Accepted for publication Sep 14, 2018.

doi: $10.21037 /$ jtd.2018.09.72

View this article at: http://dx.doi.org/10.21037/jtd.2018.09.72

In the latest few decades, the practice of "surgical therapy" has seen expansive development and been accompanied by considerable advances in minimally invasive surgery, anesthetic technique and perioperative care, in addition to a greater understanding of overall surgical pathophysiology. Accordingly, enhanced recovery after surgery (ERAS), an evidence-based multimodal protocol of perioperative care, has emerged as an inevitable outcome of the development of surgical theory and technology (1-4), and attempts to achieve the following aims: to minimize perioperative stress responses and catabolism; to decrease morbidity rates; to shorten the length of stay (LOS); to facilitate an early return to normal life through faster procedure; and to improve the prognosis of surgical patients.

Current evidence demonstrates that morbidity rates, LOS, and healthcare costs, are the 3 most common evaluation criteria for a standardized ERAS program (5-7). The earlier ERAS studies paid much more attention to the most common and easily detected complications after general abdominal surgery (8). However, knowledge of these common morbidities may not be able to offer a full picture of a variety of medical and surgical complications, which differ hugely in the disease severity and corresponding therapeutic options. Therefore, in recent years, the great majority of relevant evidence has advocated the Clavien-Dindo classification system, an effective scoring system that has been validated to distinguish the degrees of postoperative complications, for the purpose of evaluating the efficacy of an ERAS program $(9,10)$.
The summarized data from a recent evidence-based review of 38 randomized controlled trials (RCTs) indicate that the implementation of an ERAS protocol can significantly decrease the morbidity rates across surgical specialties (8). Furthermore, a validated ERAS pathway can also significantly shorten the LOS and accelerate surgical patients return to normal life, although some of these patients may further suffer from several complications needing medical intervention outside the hospital, resulting in an increased readmission rate (2-4). In the latest systematic meta-analysis conducted by our research team, we synthesized the outcome data from 7 RCTs addressing the ERAS adoption in lung cancer surgery and found a similar clinical superiority of an ERAS program for the effective control of postoperative morbidity and LOS (1).

However, as Rogers et al. (4) have recently suggested, it is still worth considering whether the clinical advantages from an ERAS program are a consequence of significant improvement in one or more individual care elements, or a consequence of the aggregation of marginal gains throughout the pathway. Therefore, in the Dr. Rogers's report, the authors aimed to identify the predictive roles of their individual ERAS elements for 30-day morbidity and prolonged LOS in patients undergoing lung resection for primary lung cancer (4). They performed a prospective cohort study based on their large institutional database of a standardized ERAS protocol and found that the age, lobectomy or pneumonectomy, $>1$ resections and delayed mobilization, could act as independent predictors for 
postoperative complications. In addition, the age, lack of preoperative carbohydrate drinks, planned high dependency unit/intensive therapy unit admission, delayed mobilization, and open approach, were also found to predict the prolonged LOS ( $>5$ days) (4).

Finally, Rogers et al. (4) indicated that a stricter adherence to the ERAS pathways could contribute to improved surgical outcomes. Several elements within an ERAS program, such as early mobilization and minimally invasive surgery, appear to play a more influential role than others (4). They suggested that a well-designed ERAS pathway should be considered for all patients undergoing lung cancer surgery. We congratulate Dr. Rogers and his colleagues for this excellent high-quality investigation on the current subject of fast-track lung cancer surgery. While, from the perspective of our institutional work, we think that the following four aspects regarding Dr. Rogers's findings may need to be further discussed.

\section{Preoperative airway preparation}

Rogers et al. (4) suggest that smoking status does not influence the admission stage in an ERAS pathway. This finding differs from our institutional evidence showing a clinical significance of smoking history before surgery. On the basis of our current RCTs, we have concluded that the pulmonary rehabilitation strategies for active smokers should include (11-15):

(I) quit smoking: cigarette cessation lasting $>2$ weeks, preferably for 4 weeks (16);

(II) short-term intensive pulmonary rehabilitation: a comprehensive pulmonary rehabilitation program before surgery, including the anti-inflammation pharmaceuticals, anti-asthma therapies, nebulized corticosteroid and bronchodilator inhalation, intensive pulmonary physiological therapy and inspiratory muscle exercise training, is highly recommended for (i) octogenarians; (ii) active smokers with a smoking index $\geq 800$ pack-year; (iii) elderly patients (age $\geq 60$ years) with a smoking index $\geq 200$ pack-year; (iv) $\geq 45$-year-old patients with a smoking index $\geq 400$ pack-year; and (v) patients who were accompanied with moderate to severe chronic obstructive pulmonary disease (COPD) (11-17).

In the Dr. Rogers's study, the authors did not provide enough details for preoperative rehabilitation or exercise strategies in the patients who were considered at high surgical risk, especially in the elderly smokers. Although age was found to serve as an independent risk factor for both postoperative morbidity and prolonged LOS in this study, we speculate that the surgical outcomes in elderly smokers may be obviously improved with a further optimization in the preoperative intensive rehabilitation regimen.

\section{Preoperative fasting}

A 12-hour fasting and 6-hour drinking prohibition before operation, with the aim of avoiding the occurrence of stomach content regurgitation with resultant aspiration pneumonia, has been widely accepted as a vital component for traditional preoperative care. However, overnight fasting may easily induce the presence of insulin resistance and additional catabolic pathways, resulting in an increased risk of some postoperative events $(18,19)$. In fact, compared to the traditional fasting policy, there has not been any compelling evidence showing a significantly higher prevalence of regurgitation, aspiration pneumonia or relevant morbidity when applying a shortened fasting for 2-3 hours. In the Dr. Rogers's study, the authors found that carbohydrate intake before surgery did not increase the 30-day morbidity rate but did significantly shorten the LOS (odds ratio: 0.57 ; $95 \%$ confidence interval: $0.36-0.96$; $\mathrm{P}=0.010$ ) (4). Their findings gave strong evidence supporting the efficacy of an unrestricted access to clear fluids for oral intake up to 2 hours and solids for oral intake up to 6 hours before the induction of anesthesia, which had been recommended by the 2013 ERAS Society Guidelines (20). Therefore, we recommend thoracic surgeons pay more attention to optimizing the fasting period and carbohydrate diets during the preoperative phase within an ERAS program.

\section{Pain control}

Perioperative pain control is generally regarded as one of key processes during the in-hospital period, and moreover, a "painless surgery" is also one of the ultimate goals of an ERAS program. In the Dr. Rogers's study, the authors reported that early mobilization was both significantly associated with a decreased 30-day morbidity and a shortened LOS (4). Indeed, we think that satisfactory pain control may play a key role in accelerating early mobilization after surgery and thus help to avoid the potential development of several fatal complications, especially deep venous thrombosis and pulmonary artery embolism (21). We agree with Rogers et al. (4) that the 
regular and combined application of acetaminophen and non-steroidal anti-inflammatory drugs (NSAIDs) is a superior analgesic regimen in an ERAS program compared to one using opiates because of the possibly severe adverse reactions induced by opiates, such as gastrointestinal disorders, respiratory depression, nausea and vomiting (22). Our institutional evidence indicates that the most productive area of pain reduction does not concern analgesics at all. In fact, according to our data, we suggest that the key areas of postoperative chest pain are located around the operative orifice for chest tube drainage and show a moderate to severe distending pain. We speculate that postoperative chest pain may be attributed to the suture fixation attached to the traditional chest tube drainage or extracorporeal ligation after chest tube removal (2). Therefore, from the perspective of preventive strategies in an ERAS program, we think that it is more appropriate and effective to optimize the current thoracic drainage system rather than to rely on too many analgesics to sufficiently ease postoperative chest pain.

\section{Postoperative pipeline management}

Our most recent study suggested that there was no necessity to use an indwelling urinary catheter during the in-hospital period for (I) the patients who have never experienced any urinary tract injury or underwent lower abdominal surgery; (II) who did not have moderate to severe prostatic hyperplasia; and (III) who were expected to receive an anesthesia time $\leq 4$ hours (23). Moreover, the optimization of chest-tube management in the most recent ERAS protocols has also dramatically shortened the LOS and days with chest-tube drainage. The chest-tube removal is no longer restricted by a 24-hour pleural drainage $>100 \mathrm{~mL}$. We are able to remove the chest tube as no air leak is detected from the chest drainage system, even though the 24-hour pleural drainage reaches up to $300 \mathrm{~mL}$ (24). In the Dr. Rogers's study, the authors permitted the pleural drainage fluids of $<500 \mathrm{~mL}$ in 24 hours as their chest tube removal criteria (4). We think this permissive protocol can be further substantiated and validated in future ERAS studies.

Given the above concerns, we think that it will be extremely essential to establish a "precise" ERAS program for individuals in lung cancer surgery (25).

First of all, an accurate clinical judgment involving an assessment of preoperative baseline characteristics (i.e., age $\geq 75$ years, active smokers with a smoking index $\geq 400$ pack-year, moderate to severe COPD, unfavorable
Charlson comorbidity index, airway hyperresponsiveness, impaired lung function, obesity, malnutrition, neoadjuvant therapy and steroid use) and procedural factors (i.e., operation time $\geq 180 \mathrm{~min}$, anesthesia time $\geq 240 \mathrm{~min}$ and possible pulmonary contusion) for the patients who are considered at significantly higher morbidity risks, should be done properly before surgery. Next, a short-term intensive pulmonary rehabilitation program is highly recommended to these high-risk patients to decrease the incidences of postoperative complications $(3,25)$.

Secondly, a specific ERAS scheme should be carried out for the patients who have obvious clinical symptoms, especially those with an obstinate cough, pain and shortness of breath, in order to alleviate the discomforts and improve their quality of life $(3,25)$.

Finally, for the asymptomatic patients without any underlying comorbidity, a process-optimized ERAS (including intraoperative warming, goal-oriented intravenous fluid therapy, avoidance of urinary catheter, early removal of chest drainage system and encouragement of mobilization within 24 hours) should be considered to improve their degree of comfort and shorten the mean $\operatorname{LOS}(3,25)$.

In summary, Rogers et al. (4) proposed that greater adherence to an ERAS pathway was significantly associated with improved surgical outcomes, indicating that several elements appear to be more influential than others. Although there are some limitations in the Dr. Rogers's study, such as the scarcity of a control group, their findings can surely put forward new insights about ERAS programs and lung cancer surgery.

\section{Acknowledgments}

We thank Stanley Crawford, from the Institution of Medical English, West China Medical Center, Sichuan University, Chengdu, China, for his help with the English language editing of this Editorial manuscript.

\section{Footnote}

Conflicts of Interest: The authors have no conflicts of interest to declare.

\section{References}

1. Li S, Zhou K, Che G, et al. Enhanced recovery programs in lung cancer surgery: systematic review and metaanalysis of randomized controlled trials. Cancer Manag 
Res 2017;9:657-70.

2. Che G. What is Enhanced Recovery After Surgery: Humanity or Technology? Zhongguo Fei Ai Za Zhi 2018;21:168-72.

3. Che G. Establishment and Optimization of Enhanced Recovery after Surgery System for Lung Cancer. Zhongguo Fei Ai Za Zhi 2017;20:795-9.

4. Rogers LJ, Bleetman D, Messenger DE, et al. The impact of enhanced recovery after surgery (ERAS) protocol compliance on morbidity from resection for primary lung cancer. J Thorac Cardiovasc Surg 2018;155:1843-52.

5. Nicholson A, Lowe MC, Parker J, et al. Systematic review and meta-analysis of enhanced recovery programmes in surgical patients. Br J Surg 2014;101:172-88.

6. Fiore JF Jr, Bejjani J, Conrad K, et al. Systematic review of the influence of enhanced recovery pathways in elective lung resection. J Thorac Cardiovasc Surg 2016;151:708-15.e6.

7. Schatz C. Enhanced Recovery in a Minimally Invasive Thoracic Surgery Program. AORN J 2015;102:482-92.

8. Kehlet H, Wilmore DW. Evidence-based surgical care and the evolution of fast-track surgery. Ann Surg 2008;248:189-98.

9. Dindo D, Demartines N, Clavien PA. Classification of surgical complications: a new proposal with evaluation in a cohort of 6336 patients and results of a survey. Ann Surg 2004;240:205-13.

10. Li P, Lai Y, Zhou K, et al. Analysis of Postoperative Complications and Risk Factors of Patients with Lung Cancer through Clavien-Dindo Classification. Zhongguo Fei Ai Za Zhi 2017;20:264-71.

11. Zhou K, Su J, Lai Y, et al. Short-term inpatient-based highintensive pulmonary rehabilitation for lung cancer patients: is it feasible and effective? J Thorac Dis 2017;9:4486-93.

12. Huang J, Lai Y, Zhou X, et al. Short-term high-intensity rehabilitation in radically treated lung cancer: a threearmed randomized controlled trial. J Thorac Dis 2017;9:1919-29.

13. Lai Y, Su J, Qiu P, et al. Systematic short-term pulmonary rehabilitation before lung cancer lobectomy: a randomized trial. Interact Cardiovasc Thorac Surg 2017;25:476-83.

14. Lai Y, Huang J, Yang M, et al. Seven-day intensive preoperative rehabilitation for elderly patients with lung cancer: a randomized controlled trial. J Surg Res 2017;209:30-6.

15. Lai Y, Su J, Yang M, et al. Impact and Effect of Preoperative Short-term Pulmonary Rehabilitation Training on Lung Cancer Patients with Mild to Moderate
Chronic Obstructive Pulmonary Disease: A Randomized

Trial. Zhongguo Fei Ai Za Zhi 2016;19:746-53.

16. Multi-disciplinary Perioperative Airway Management Group. Chinese expert consensus statement on multidisciplinary perioperative airway management (2016 version). Zhongguo Xiong Xin Xue Guan Wai Ke Lin Chuang Za Zhi 2016;23:641-5.

17. Che $G$. The clinical value of lung rehabilitation in the perioperative period of enhanced recovery after surgery. Hua Xi Yi Xue 2018;33:104-7.

18. Nygren J, Soop M, Thorell A, et al. Preoperative oral carbohydrate administration reduces postoperative insulin resistance. Clin Nutr 1998;17:65-71.

19. Sarin A, Chen LL, Wick EC. Enhanced recovery after surgery-Preoperative fasting and glucose loading-A review. J Surg Oncol 2017;116:578-82.

20. Gustafsson UO, Scott MJ, Schwenk W, et al. Guidelines for perioperative care in elective colonic surgery: Enhanced Recovery After Surgery (ERAS(®)) Society recommendations. World J Surg 2013;37:259-84.

21. Che G. Evidence and Practice of Enhanced Lung Recovery after Surgery in Patients Undergoing Lung Surgery. Zhongguo Fei Ai Za Zhi 2017;20:371-5.

22. Kehlet H. Enhanced Recovery After Surgery (ERAS): good for now, but what about the future? Can J Anaesth 2015;62:99-104.

23. Qiu F, Yang M, Che GW, et al. Risk factors of postoperative urinary retention without perioperative urinary catheterization after video-assisted thoracoscopic surgery lobectomy. ZhongGuo Xiong Xin Xue Guan Wai Ke Lin Chuang Za Zhi 2016;23:328-33.

24. Xie HY, Xu K, Tang JX, et al. A prospective randomized, controlled trial deems a drainage of $300 \mathrm{ml} /$ day safe before removal of the last chest drain after video-assisted thoracoscopic surgery lobectomy. Interact Cardiovasc Thorac Surg 2015;21:200-5.

25. Che G, Liu L. Enhanced Lung Recovery after Surgery, Is It A Necessary for Precision Therapy? Zhongguo Fei Ai Za Zhi 2017;20:549-54.

(English Language Editor: John Gray, AME Publishing Company)

Cite this article as: Li S, Che G, Shen C, Zhou K. Current situation and consideration on the enhanced recovery protocols in lung cancer surgery. J Thorac Dis 2018;10(Suppl 33):S3855S3858. doi: $10.21037 /$ jtd.2018.09.72 